\title{
The development and application of an original 3D laser scanning: a precise and nondestructive structural measurements system
}

\author{
Kexin Li, Jun Wang \\ College of Civil Engineering, Northeast Forestry University, Harbin 150000, China \\ like@nefu.edu.cn, bttp://orcid.org/0000-0003-3854-1710 \\ jun.w.619@163.com
}

\section{Dawei Qi}

School of Science, Northeast Forestry University, Harbin 150000, China

qidw9806@126.com

\begin{abstract}
Recently, a number of Non-Destructive Testings (NDTs) equipment which can partially replace human-conducted on-site inspections has been implemented for detecting modern civil architectural structures. However, the situation of implementing 3D laser scanning measurement technology worldwide is not optimistic: several inconvenient flaws are troubling users (e.g., heavy, costly, hard to move, hard to inspect and etc.). Therefore, a new equipment to address the problems is urgently demanded. A 3D laser scanning system is designed composed of high precision elevating platform and small 2D laser ranging sensor. Manufacture Process of the 3D laser scanning system is described in detail. A comparative analysis with other non-contact measurement experiments based on the bending fatigue load test, the scanning designed efficiency and feasibility has been proved. The information of micro-damage and depth of the structure can be quickly captured by the designed 3D laser scanning system. Its detecting performance is better than the traditional methods. To overcome the challenges of the 3D laser scanning on-site measurement technology, this paper proposes the manufacture process of the 3D scanning system with a high precision, miniaturization and lightweight.
\end{abstract}

KEYWORDS: Structure detection; 3D laser scanning; Nondestructive testing; Digital image process; Measurement on site.

\section{open ACCESS}

Citation: Li, K., Wang, J., Qi, D., The development and application of an original 3D laser scanning: a precise and nondestructive structural measurements system, Frattura ed Integrità Strutturale, 51 (2020) 386-397.

Received: 15.10 .2019

Accepted: 01.12.2019

Published: 01.01 .2020

Copyright: (C) 2020 This is an open access article under the terms of the CC-BY 4.0, which permits unrestricted use, distribution, and reproduction in any medium, provided the original author and source are credited.

\section{INTRODUCTION}

$\mathrm{B}$ uilding structure testing can provide a reliable basis for the quality assessment of engineering structures, the identification of existing structural properties and the verification of structural calculation theory [1]. The building structure is inspected on site through various measurement techniques. The structural related parameters are obtained. The effective feature information reflecting the current state of the structure is captured. And the relationship 
between the structure and the resistance is analyzed. It is used to promote the comprehensive judgment and evaluation of the safety and reliability of the structure. [2]. However, the detection process is limited by the complex constraints of the engineering site. The traditional structure detection technology cannot fully meet the requirements of modern building structure detection in terms of measuring dimensions, range and frequency [3]. For example, the Progressive Collapse study, which has received extensive attention from the international civil engineering community. The dynamic response of the structure is triggered by the initial initialization of the test component's own components, resulting in high frequency vibration and large deformation. The dynamic strain and the displacement are required to be measured, which exceed the application range of traditional measurement technology [4]. Therefore, it is very necessary to develop precision detection devices and related measurement technologies in combination with various emerging fields. Thereby, the non-destructive detection and reliability identification of modern building structures can be realized efficiently [5].

As one of the latest advances in the field of structural measurement, non-contact measurement technology has the advantages of undamaged, accuracy and objectivity [6]. Among them, Digital Image Correlation (DIC) has been widely used in structural detection experiments [7]. However, DIC technology requires higher illumination conditions for the detection environment. It generally requires multiple devices to work together to obtain spatial deformation information [8]. And the 3D laser scanning measuring device has the following advantages: 1) non-destructive: getting rid of installing the sensor on the object to be detected; 2) strong environmental adaptability: the laser itself can be used as a light source to resist interference; 3) efficient detection: can achieve 3D information acquisition of a single device [9-12]. Therefore, the 3D laser scanning technology have lately received great attention in the field of building structure inspection [13, 14]. Park [15] applied 3D laser scanning technology to structural health monitoring, and verified the effectiveness of the method based on the static loading test of simply supported steel beams. Olsen [16] used 3D laser scanning technology to quantitatively describe the structural damage based on the reinforced concrete frame joint test. Dai [17] obtained the 3D laser scanning data of the steel beam structure and extracted the deformation field information of the structure.

The widespread application of commercial, integrated 3D laser scanners to structural testing are facing numerous challenges: 1) Scanners are usually expensive, bulky, and complex; 2) The detection environment is limited, and it is difficult for the inspectors to operate flexibly; 3) Usually, the scanner scans large structures, and it is difficult to accurately construct a three-dimensional model of the structural test object. Therefore, it is worthwhile devoting much effort to develop a 3D laser scanning device that is miniaturized, lightweight, high precision and low-cost, thereby achieving accurate modeling of modern structural tests [18]. The laser ranging sensor is characterized by accuracy and efficiency. Therefore, it plays an important role in measurement, navigation and security [19]. Among them, the 2D laser ranging sensor can form a single complete scanning plane by single line scanning. We can independently design a 3D scanning device that drives the 2D laser sensor to move up and down according to the needs of the detection object and the environment.20]. The designed scanning device is more adaptable and flexible, can accurately construct a 3D model based on lower cost. The 3D scanning device integrated by 2D laser sensor has been widely used in environmental modeling, mechanical automation and other fields. For example, the Fraunhofer Institute for Autonomous Intelligent Systems in Germany developed an autonomous mobile robot with a 2D laser range finder and successfully applied it to 3D exploration and digital simulation for indoor environments [21]. Chou [22], etc., installed a 2D laser sensor on a rotating four-bar linkage to create an indoor 3D mapping automatic scanning robot. Cai Jun [23] developed a 3D laser scanning system consisting of a high-precision rotating head and a small 2D laser ranging sensor to achieve high-precision automation environment modeling. The detection technology of 3D laser scanning by adding 2D laser sensor to the 1D transmission actuator has achieved good research results [24-25]. However, despite these considerable advantages, this technology has not been widely promoted in structural testing studies.

In summary, it is essential to independently develop a 3D laser scanning system for modern structural inspection. The stepper motor drives the fine movement of the sliding group to realize the lifting and scanning of the two-dimensional laser ranging sensor. This system is portable, more accurate, more adaptable and less costly. It realizes non-destructive, efficient and accurate detection of structural damage 3D information. Based on the RPC bending fatigue test, the reliability of the 3D modeling and structural damage information measurement data of the device was verified.

\section{D LASER SCANNING SYSTEM DESIGN}

Principles of System Design

he 3D laser scanning system designed in this paper is mainly composed of a $2 \mathrm{D}$ laser ranging sensor, a sliding mold combination, a bracket, a control box and a computer, as shown in Figure 1. The scanning system is divided into four parts: a transmission actuator, a transmission control device, a laser ranging sensor, and a sensor control 
interface. The transmission actuator is driven by a stepping motor to output a high-precision, controllable lifting movement. The drive control unit consists of a stepper motor driver, PLC and related components. The control slider performs a uniform linear ascending and descending motion on the slide rail through the M programming language. In order to control the uniform linear lifting scanning action of the laser ranging sensor, it is fixedly mounted on the slider through the connecting plate. The sensor scan plane is parallel to the ground and perpendicular to the drive direction. The parameters of the laser sensor are set on the surface of the sensor control interface, so that the scanning optical center lifting speed is consistent with the linear sliding group motion output.

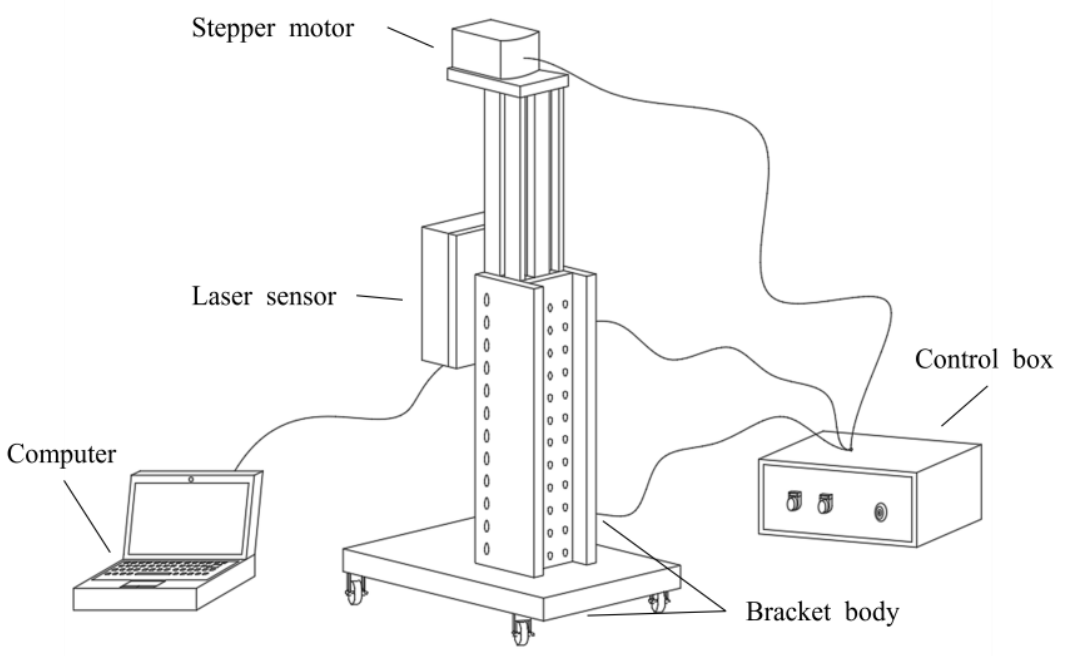

Figure 1: Design drawing of 3 d laser scanning system.

\section{System measurement principle}

Two coordinate systems for the structural characteristics of the 3D laser measurement system are defined as followed: 1) The coordinate system of laser ranging sensor position: $\left.\left\{\mathrm{O}_{\mathrm{A}}-\mathrm{X}_{\mathrm{A}} \mathrm{Y}_{\mathrm{A}} \mathrm{Z}_{\mathrm{A}}\right\} ; 2\right)$ The coordinate system of the image acquired by laser scanning: $\{\mathrm{O}-\mathrm{XYZ}\}$. The origin of the $\left\{\mathrm{O}_{\mathrm{A}}-\mathrm{X}_{\mathrm{A}} \mathrm{Y}_{\mathrm{A}} \mathrm{Z}_{\mathrm{A}}\right\}$ coordinate system is located at the sensor transmission port, $\mathrm{X}_{A} \mathrm{O}_{A} \mathrm{Y}_{\mathrm{A}}$ is parallel to the scanning surface of the two-dimensional laser, and $\mathrm{Z}_{\mathrm{A}}$ is the transmission direction. The moving speed of the laser sensor in the $\mathrm{Z}$ direction is $\mathrm{v}(\mathrm{m} / \mathrm{s})$, and the movement time is $t(\mathrm{~s})$. In the initial state, the laser emitting end (red circle) coincides with the origin. The angle between the laser emitted by the transmitting end and $\mathrm{X}_{\mathrm{A}}$ is $\alpha$, and the coordinate of the intersection with the object to be detected is $\mathrm{S}\left(\mathrm{x}_{\mathrm{a}}, \mathrm{x}_{\mathrm{b}}, \mathrm{X}_{\mathrm{c}}\right)$. The laser is emitted to the object, and the reflected light on the object is reflected to the receiving end (green circle). The angle between the emitted light and the reflected light is $\theta$. The $\mathrm{X}$-axis of the $\{\mathrm{O}-\mathrm{XYZ}\}$ coordinate system is parallel to the intersection of the scanning plane and the detected object. The $\mathrm{Y}$-axis is parallel to the scanner drive direction. The $\mathrm{Z}$-axis represents the distance from the laser emitting end of the scanning plane to the detected object.

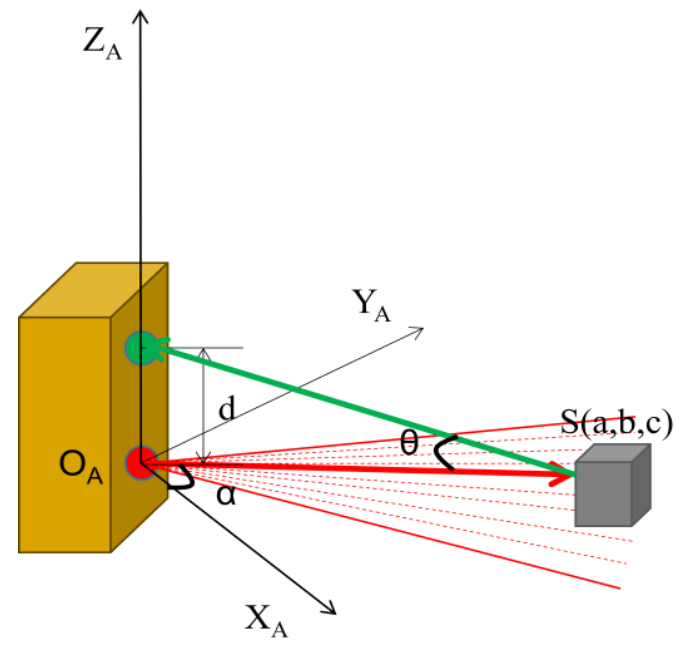

Figure 2: Laser ranging sensor coordinate system $\left\{\mathrm{O}_{\mathrm{A}}-\mathrm{X}_{\mathrm{A}} \mathrm{Y}_{\mathrm{A}} \mathrm{Z}_{\mathrm{A}}\right\}$ 
In the $\left\{\mathrm{O}_{\mathrm{A}}-\mathrm{X}_{\mathrm{A}} \mathrm{Y}_{\mathrm{A}} \mathrm{Z}_{\mathrm{A}}\right\}$ coordinate system, the three-dimensional coordinates of $\mathrm{S}\left(\mathrm{x}_{\mathrm{a}}, \mathrm{x}_{\mathrm{b}}, \mathrm{x}_{\mathrm{c}}\right)$ are expressed as follows:

$$
\left\{\begin{array}{l}
x_{a}=\rho \cos \alpha \\
y_{a}=\rho \sin \alpha \\
z_{a}=v t
\end{array}\right.
$$

The distance from the sensor to the surface of the object can be expressed by Equation (2):

$$
\rho=d / \tan \theta
$$

Therefore, the position coordinates of any light spot $\mathrm{S}$ can be expressed as:

$$
\left\{\begin{array}{l}
x_{a}=d \cos \alpha / \tan \theta \\
y_{a}=d \sin \alpha / \tan \theta \\
z_{a}=v t
\end{array}\right.
$$

Then the laser scanned image is obtained and establish the coordinate system $\{\mathrm{O}-\mathrm{XYZ}\}$ is established, as shown in Fig. 3.

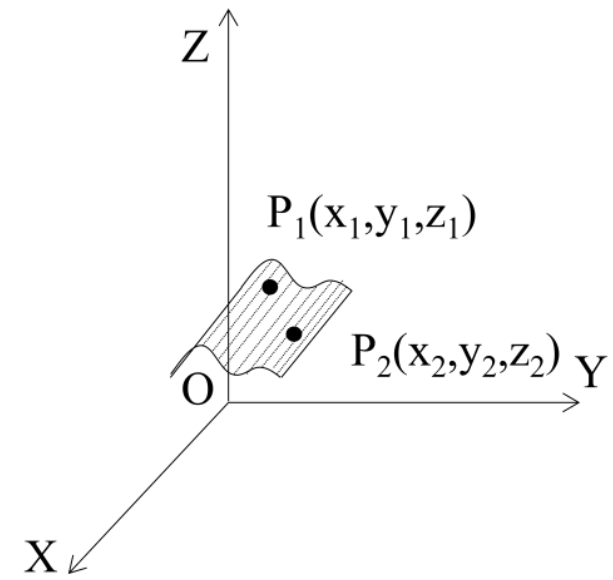

Figure 3: Laser scanning image coordinate system $\{\mathrm{O}-\mathrm{XYZ}\}$.

The laser scanned image is an object model that is recombined by laser spots. Therefore, the detection data of the structure can be acquired by the position change information of any two points $\mathrm{P}_{1}\left(\mathrm{x}_{1}, \mathrm{y}_{1}, \mathrm{z}_{1}\right)$ and $\mathrm{P}_{2}\left(\mathrm{x}_{2}, \mathrm{y}_{2}, \mathrm{z}_{2}\right)$ on the image. The deformation information of the structure can be calculated by the formula (4).

$$
P_{2} P_{1}=\sqrt{\left(x_{2}-x_{1}\right)^{2}+\left(y_{2}-y_{1}\right)^{2}}
$$

where $y=\mathrm{vt}, \mathrm{x}$ is related to the variables $\alpha, \theta$ and the size of the detected object.

The variation of the structure elevation information can be obtained by formula (5).

$$
D=\left|z_{2}-z_{1}\right|
$$

where $z=d / \tan \theta$.

Therefore, the established 3D laser scanning system can detect structural damage by acquiring the positional changes of the spots on the laser scanned image. 


\section{IMPLEMENTATION OF 3D LASER SCANNING SYSTEM}

\section{Bracket}

he bracket is used to support and stabilize the transmission actuator, including the main body of the bracket, the base and the wheel. The overall composition is shown in Figure4.

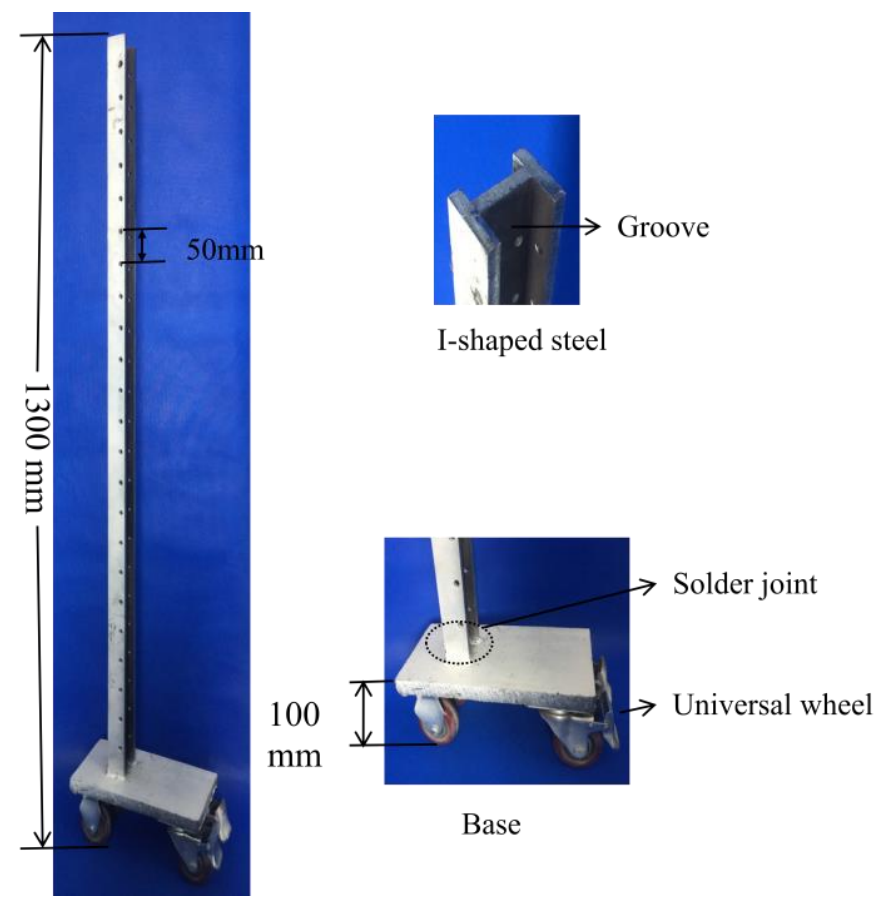

Figure 4: Bracket composition.

The main body of the bracket is made up welded I-shaped steel with a total length of $1200 \mathrm{~mm}$. The overall height of the bracket is $1300 \mathrm{~mm}$. In order to facilitate the installation and movement of the sliding group, a hole is placed on each side of the struts $50 \mathrm{~mm}$ apart, for a total of 23 holes. The maximum effective detection height is $2000 \mathrm{~mm}$, and can be adjusted arbitrarily according to the on-site inspection environment. In order to ensure good stability and portability of the struts, the struts and the base are fixed by welding. The pulleys are installed on the four corners of the base.

\section{Sliding module}

The combination of sliding mode is as the basic support of the transmission system, including the strut, stepper motor, slider, screw, as shown in Figure 5.

In order to ensure a perfect nested connection between the sliding group and the bracket body, M4 nut slots corresponding to the bracket body are disposed on both sides and the rear of the pillar. For the purpose of improving module accuracy, a $1200 \mathrm{rpm}$ stepper motor and a grade C7 \& G1610 ball screw are provided. The slider is a rectangular parallelepiped with a threaded hole in the center that can pass through the screw.

\section{Connection and fixation}

There is a nested connection between the bracket and the sliding group. There is a fixed connection between the stand and the scanner. These two types of connections directly affect the scanning accuracy of the entire drive system. In order to ensure accurate nesting connection between the bracket and the sliding group, the drilling of the nested connection is set, and the specific connection manner is shown in Figure. 6(a). For the purpose of calibrating the flatness of the back of the laser sensor, a connecting plate in which the laser sensor is fixedly connected to the slider is fabricated. The four connection holes in the middle of the connecting plate are subjected to countersinking treatment, and the specific connection form is shown in Figure. 6(b). 


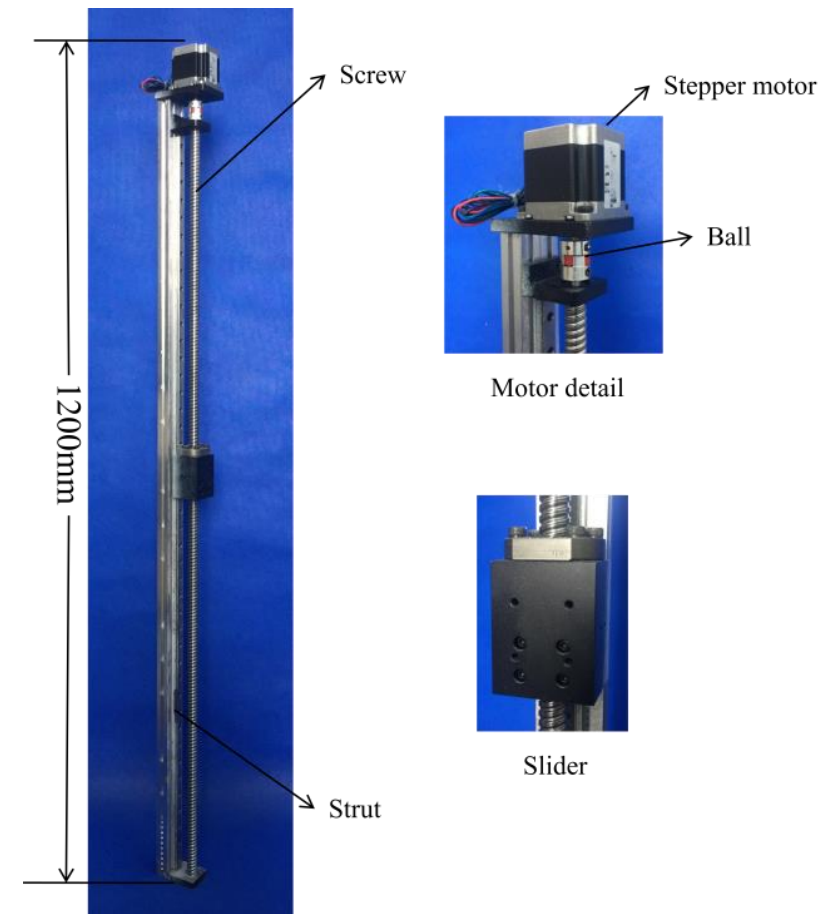

Figure 5: Slide group detail.
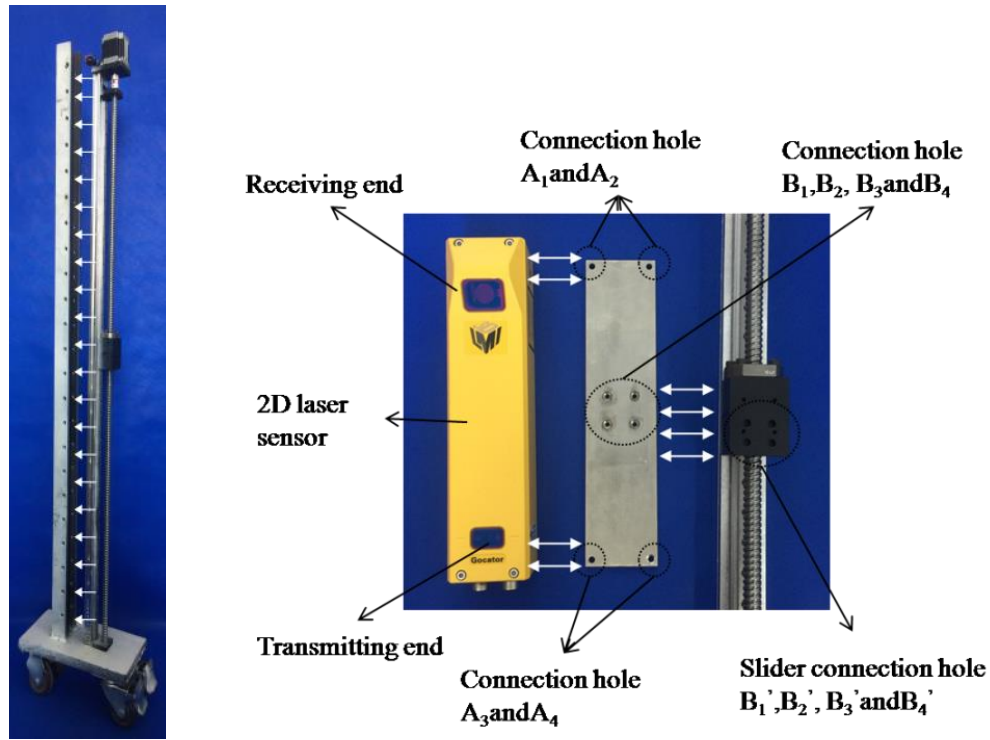

(a) Nested loops join

(b) connecting the fixed

Figure 6: Connection and fixation.

\section{Control box}

The transmission control device composed of the control box and its internal components is shown in Figure 7. In order to control the operation of the transmission system, the built-in transformer of the control box provides a regulated power supply for the stepper motor. The controller sends a stable pulse signal to the driver, which converts the signal to an angular displacement and transmits it to the stepper motor. A button capable of controlling the direction of the driver is provided outside the cabinet. 


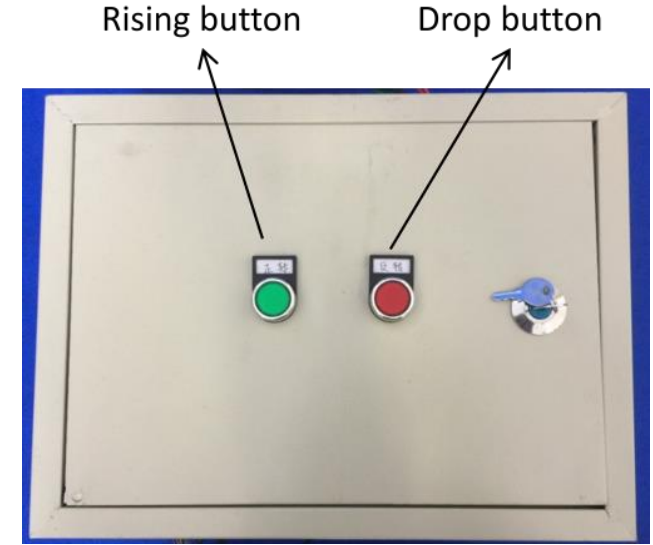

(a) External

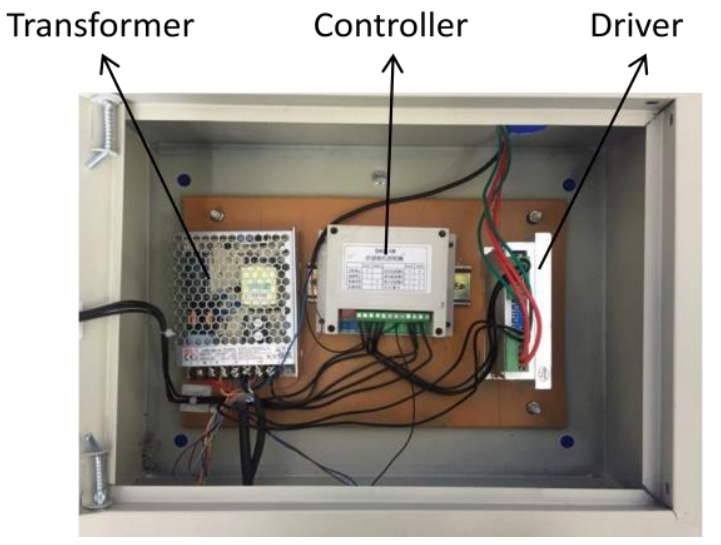

(b) internal

Figure: 7 Detail of the drive actuator.

\section{Parameter setting and automatic data collection}

The Gocator-2080 laser sensor is connected to the power supply in the control box via a 19-pin cable, connected to the computer by the M12 cable. The scanning speed, exposure and other parameters are set on the laser ranging sensor setting interface. Figure 8 shows a full view of the 3D laser scanning system. The position of the main body of the stent is adjusted, the structure to be inspected in the effective area of the scan is placed, and data automatically is collected. The $3 \mathrm{D}$ scan information of the detected object are obtained. And the data storage is completed .

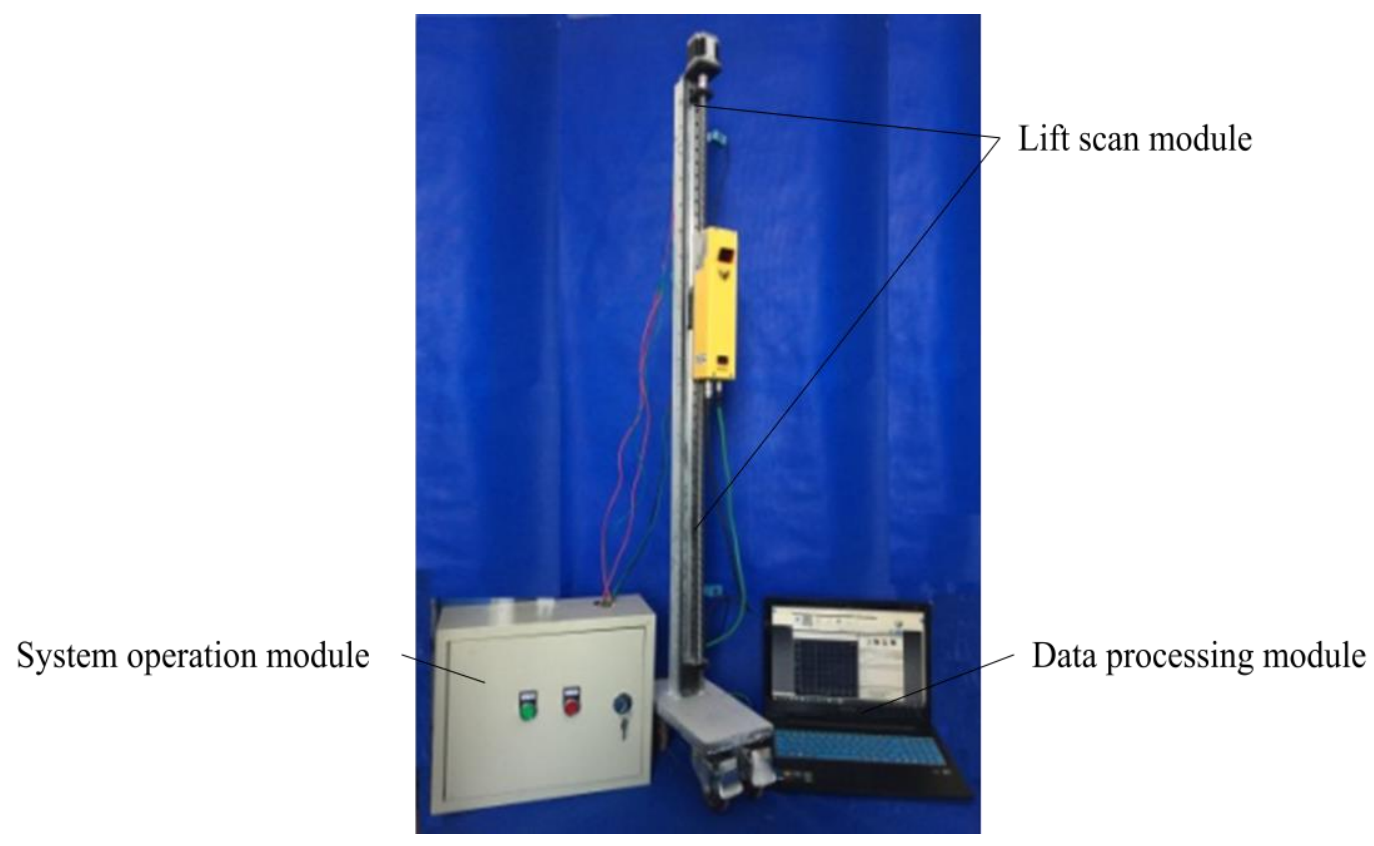

Figure 8: 3D laser scanning system.

\section{Application}

In order to fully verify the reliability and practicability of the structure detection of the 3D laser scanning system. Based on the concrete bending fatigue test, the concrete structure damage detection is carried out. It is the first time, the 3D laser scanning system was used to detect fatigue damage of the structural.

- Automatic data collection

The structure is located in the scanning sector detection area by adjusting the horizontal position of the holder body. In order to ensure complete structural modeling information, the effective detection range in the vertical direction is greater than the height of the detected object. Figure 9 shows the data acquisition process. 


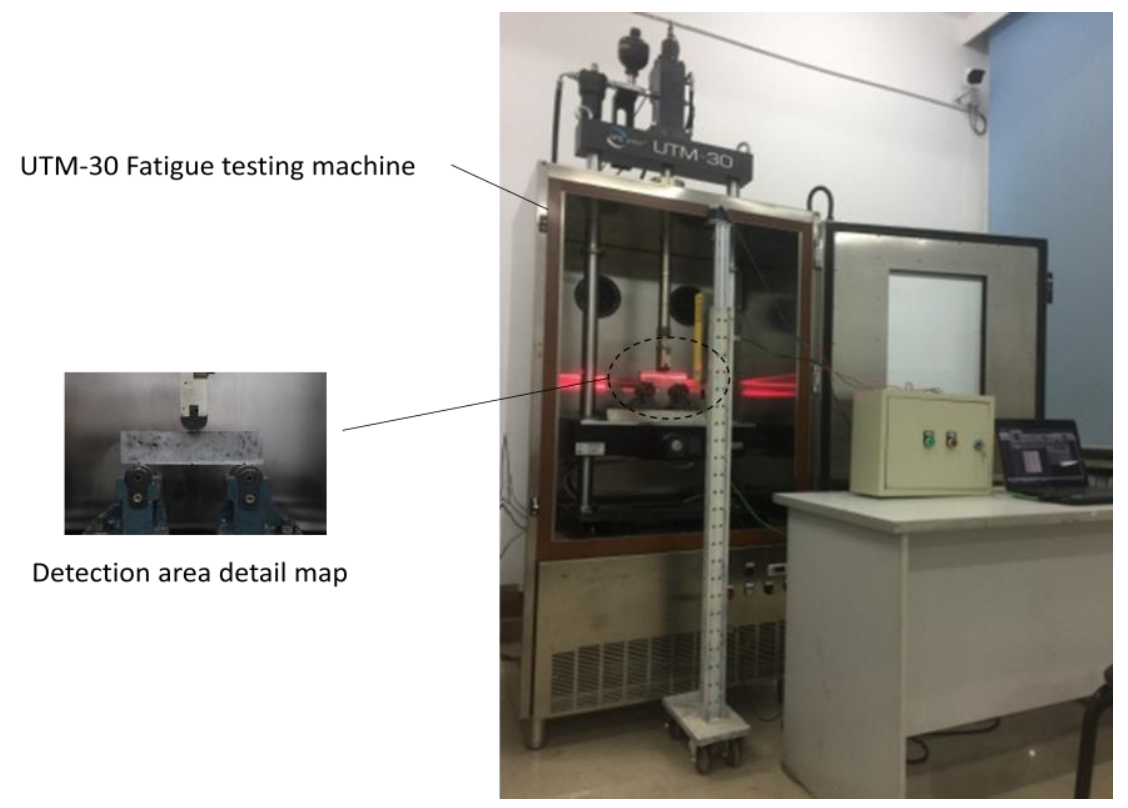

Figure 9: Data acquisition.

The data of the damaged area can be automatically collected through the buttons on the control box.

- 3D Image efficient acquisition

Figure 10 shows a scanned point cloud image of the structure acquired in the $\{\mathrm{O}-\mathrm{XYZ}\}$ coordinate system. In order to compare the measurement accuracy of the 3D laser scanning system, based on the bending fatigue loading test, 100 laser scanning experiments were performed for each group of structural tests.

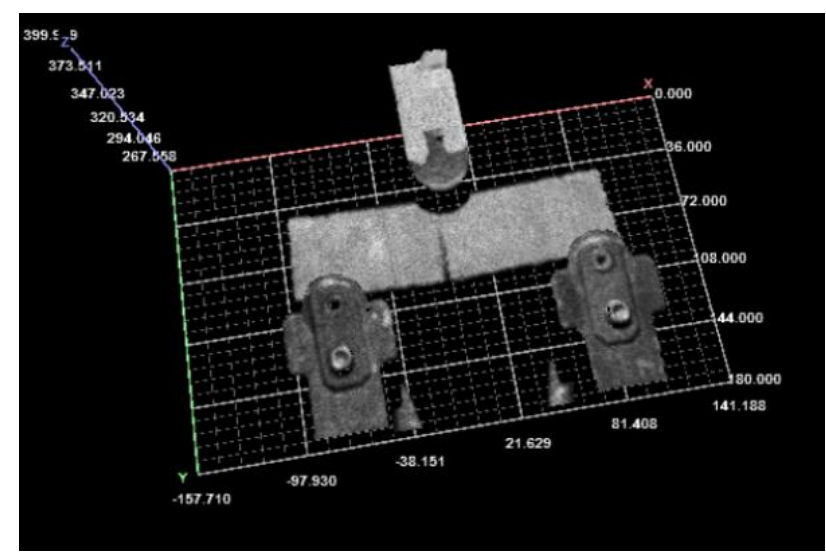

Figure 10: Structure point cloud diagram.

The 3D image of the damaged area is quickly displayed on the computer interface with an average efficiency of 3s per image. The performance of the scanning system was verified. Based on this experiment, the performance of the scanning system was verified. The high-frequency vibration of the detection object can be neglected, the scanning precision is high, and the scanning speed is fast; damage information on the plane and space of the damaged area can be obtained, and the damage situation can be more comprehensively analyzed; the position of the scanner can be arbitrarily adjusted according to the conditions of the on-site inspection site, which is convenient for movement; it is not affected by environmental factors of the detection site, and has strong adaptability.

The scanning effective area is a fan-shaped space of 350-750 $\mathrm{mm}$ from the scanning sensor with an accuracy of $0.005 \mathrm{~mm}$. The scanning accuracy is high, but the area is small. It is not suitable for scanning large structures. This problem can be improved by replacing the model of the scanning sensor. The effective height of the scan is affected by the length of the slide group. The improvement of this problem can be achieved by increasing the length of the sliding group to meet the engineering structure detection needs. 


\section{RESULTS AND DISCUSSION}

\section{Defect detection}

he structure contains depressions, holes, and crack defects caused by fatigue loading. The Region of Interest (ROI) of each defect point cloud image is extracted, and the defect detection is performed according to the formula (4) and the formula (5), as shown in Figure 11.

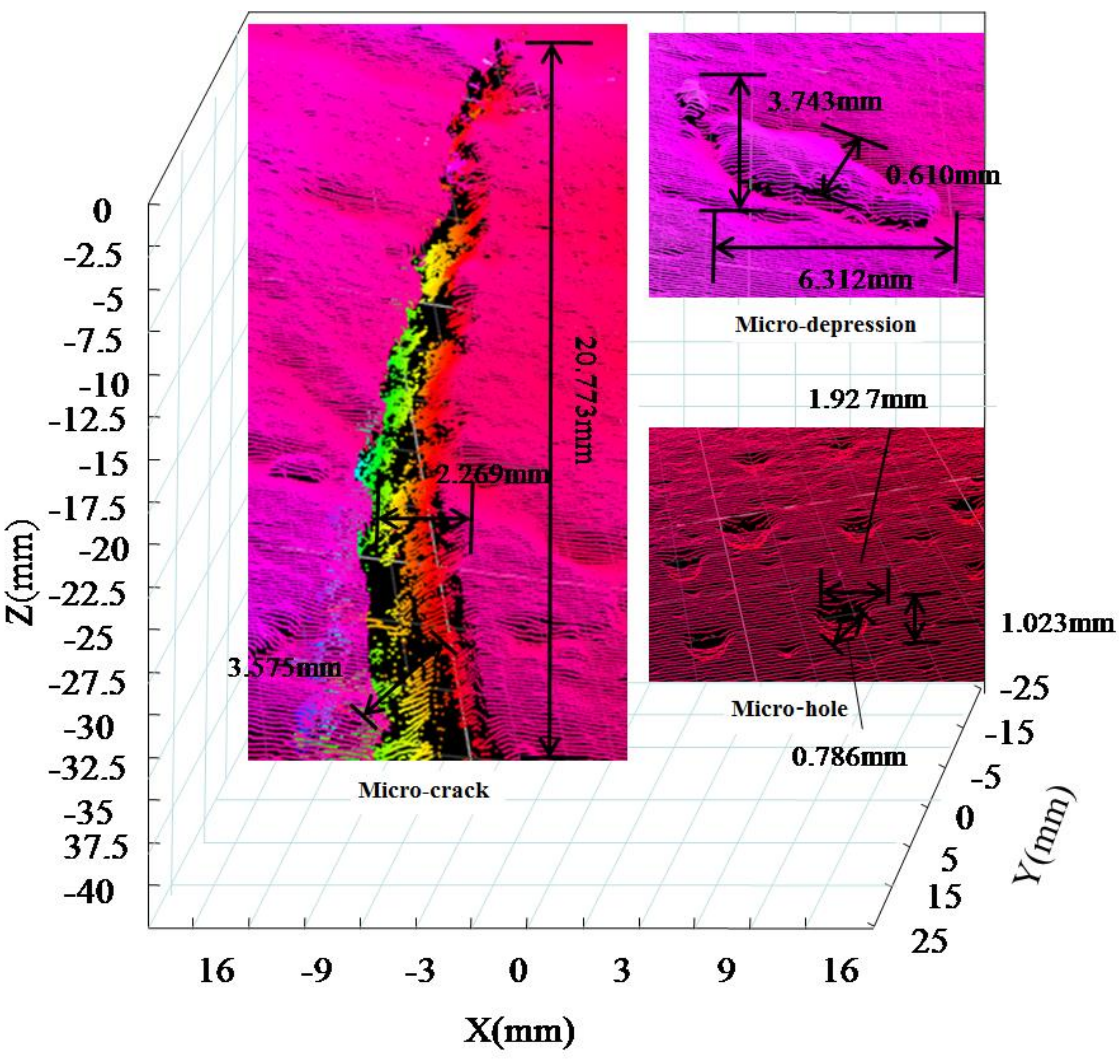

Figure 11: Defect detection result.

The specific detection results of each defect are marked in Figure.11, including the size and elevation data information.

\section{Analysis of results}

The measurement results are obtained based on Digital Image Correlation (DIC) technology and the yernier caliper detection method based on traditional detection technology respectively. The test results and analyze test performance is compared. The detection method based on DIC technology needs sufficient illumination. Moreover, structural damage information needs to be obtained in conjunction with digital image correlation techniques. And the conventional measurement method needs a professional inspector. The scanning device designed in this study is consistent with the defect detection results obtained by each detection means. The rationality and reliability of the system are fully proved. At the same time, the scanning device designed in this study is superior to other detection technologies in performance. The comparison of performance as shown in Table 1.

The data in Table 1 shows that the traditional measurement method and the flaw detection instrument combined with DIC have higher requirements on the light source, lower objectivity and efficiency, and it is difficult to capture the elevation information of the defect. The 3D laser scanning system developed in this paper realizes the modeling of the measurement structure. It can achieve more accurate detection results under any condition. And it has the advantages of wide measurement range and high accurate of detection. It has no blind spot area and high detection efficiency. In this comparative analysis experiment, the 3D laser scanning system showed stronger environmental adaptability and higher detection efficiency. The developed scanning system has high scanning accuracy and fast scanning speed. It can 
comprehensively analyze the changes of damage in the plan and space. And it is not affected by the environmental factors of the test site and has high adaptability.

\begin{tabular}{cccccc}
\hline Type & Condition & Accuracy $(\mathrm{mm})$ & Range & Efficiency $(\mathrm{s})$ & Depth \\
Gocator2080 & -- & $\pm 0.005 \mathrm{~mm}$ & $\begin{array}{c}\text { Level: } 0-120^{\circ} \\
\text { Vertical: } 0-2000 \mathrm{~mm}\end{array}$ & 3 & Available \\
HCCK102 & light source & $\pm 0.01 \mathrm{~mm}$ & $0-8 \mathrm{~mm}$ & 45 & - - \\
Paola5385 & Professional & $\pm 0.03 \mathrm{~mm}$ & $0-150 \mathrm{~mm}$ & 60 & - - \\
\hline
\end{tabular}

Table 1: Measurement performance comparison.

\section{CONCLUSIONS}

his paper developed a 3D laser scanning structure test measuring device independently, which can widely adapt to the testing conditions of modern structural tests. The 3D laser scanning system consist of a linear slide group and a $2 \mathrm{D}$ laser ranging sensor. The stepping motor controls the fine movement of the sliding group screw to realize the lifting and scanning of the laser ranging sensor. The following conclusions were made:

1) The $3 \mathrm{D}$ coordinate system of the laser scanning measuring device is established, and the calculation formula for detecting the structure size and elevation data information is obtained.

2) The whole process of developing the scanning system is summarized, including the construction of the bracket system, the construction of the transmission device, the simulation of the control device and the connection and fixing of various parts.

3) Based on the RPC bending fatigue test, the scanning platform of this experiment was compared with the traditional method and DIC technology to verify the reliability and superiority of the operating system.

The results show that the system achieves 3D modeling of structural inspection objects compared to other detection techniques. It has stronger environmental adaptability, higher measurement accuracy and lower cost. The developed 3D laser scanning system can overcome the constraints of environmental constraints and hardware equipment, and realize the efficient and accurate measurement of modern structural tests.

\section{ACKNOWLEDGMENTS}

$\mathrm{T}$

his work was supported by General program of the Natural science foundation project of Heilongiiang province (ZD2019E001), National natural science foundation of China (51778186) and Heilongjiang post-doctoral research foundation project (LBH-Q 15011).

\section{REFERENCE}

[1] Cha, Y. J., Buyukozturk, O. (2015). Structural Damage Detection Using Modal Strain Energy and Hybrid Multiobjective Optimization. Computer-Aided Civil and Infrastructure Engineering, 30(5), pp. 347-358.

[2] Abdeljaber, O., Avci, O., Kiranyaz, S., et al (2017). Real-time vibration-based structural damage detection using onedimensional convolutional neural networks. Journal of Sound \& Vibration,388, pp.154-170.

[3] Zhao, L., et al. (2017). A review on measurement technology for structure in civil engineering. Journal of Xi'an University of Architecture \& Technology (Natural Science Edition.49, pp. 48-55.

[4] Daigoro, I. (2018). Progressive Collapse Analysis of Structures. Numerical Codes and Applications, 12(2), pp.59-66.

[5] Zhao, X., Li, Q. (2017). A review on measurement technology for structural testing in civil engineering. J. xi'an Univ. of Arch. \&. Tech. (Natural Science Edition), 49(01), pp. 48-55 
[6] Chen, C., Li, Q, Yan, Q. (2018). Mobile robot simultaneous localization and mapping based on heterogeneous sensor information fusion. Science Technology and Engineering, 18 (13), pp.86-91

[7] Shang, M. Y., Weizhong, C., Diansen, Y. (2017). Experimental study of brittle rock failure based on three-dimensional digital image correlation technique. Rock and Soil Mechanics, 38(01), pp.117-123

[8] Pan, B., Qian, K., Xie, H. (2009). Two-dimensional digital image correlation for in-plane displacement and strain measurement: a review. Measurement Science \& Technology, 20(6), pp. 062001

[9] Yan, T., Wang, X., Zhu, H., et al (2018). Evaluation of Object Surface Edge Profiles Detected with a 2-D Laser Scanning Sensor. Sensors, 18(11).

[10] Niclass, C., Rochas, A., Besse, P. A., et al. (2005). Design and characterization of a CMOS 3-D image sensor based on single photon avalanche diodes. IEEE Journal of Solid-State Circuits, 40(9), pp. 0-1854.

[11] Monserrat, O., Crosetto, M. (2008). Deformation measurement using terrestrial laser scanning data and least squares 3D surface matching. ISPRS Journal of Photogrammetry and Remote Sensing, 63(1), pp. 142-154.

[12] Zhe, C., Fumin, Z., Xinghua, Q., et al (2015). Fast Measurement and Reconstruction of Large Workpieces with Freeform Surfaces by Combining Local Scanning and Global Position Data. Sensors, 15(6), pp. 14328-14344.

[13] Nagarajaiah, S., Yang, Y. (2017). Modeling and harnessing sparse and low rank data structure: a new paradigm for structural dynamics, identification, damage detection, and health monitoring. Structural Control \& Health Monitoring, 24(1).

[14] Yong-Shang, M. A., Wei-Zhong, C., Dian-Sen, Y., et al (2017). Experimental study of brittle rock failure based on three-dimensional digital image correlation technique. Rock \& Soil Mechanics.

[15] Park, H. S., Lee H. M., Adeli, H., et al (2007). A New Approach for Health Monitoring of Structures: Terrestrial Laser Scanning. Computer-Aided Civil and Infrastructure Engineering, pp. 22(1), 19-30.

[16] Olsen, M. J., Kuester, F., Chang, B. J., et al (2010). Terrestrial Laser Scanning-Based Structural Damage Assessment. Journal of Computing in Civil Engineering, 24(3), pp. 264-272.

[17] Dai, et al (2017). Application of gray information in LiDAR scan point cloud data for structural deformation field calculation. Journal of Building Structures. 38 (3), pp. 151-157.

[18] Aytekin, C., Rezaeitabar, Y., Dogru, S., et al (2015). Railway Fastener Inspection by Real-Time Machine Vision. IEEE Transactions on Systems Man \& Cybernetics Systems, pp. 45(7), 1101-1107.

[19] Pejic, M., Ogrizovic, V., Bozic, B., et al (2014). A simplified procedure of metrological testing of the terrestrial laser scanners. Measurement, 53, pp. 260-269.

[20] Soudarissanane, S. S., Lindenbergh, R. C. (2012). Optimizing terrestrial laser scanning measurement set-up. ISPRS International Archives of the Photogrammetry, Remote Sensing and Spatial Information Sciences

[21] Surmann, H., Nüchter, A., Hertzberg, J. (2003). An autonomous mobile robot with a 3D laser range finder for 3D exploration and digitalization of indoor environments. Robotics and Autonomous Systems, 45(3-4), pp. 181-198.

[22] Chou, Y. S., Liu, J. S. (2013). A Robotic Indoor 3D Mapping System Using a 2D Laser Range Finder Mounted on a Rotating Four-Bar Linkage of a Mobile Platform. International Journal of Advanced Robotic Systems, pp.10.

[23] Cai, et al (2018). A 3D laser scanning system design and parameter calibration. Journal of Beijng University of Aeronautics and Astronautics, 44 (10), pp.2208-2216

[24] Yongkun, W., Ju, H., Xingshun, W. (2014). A real-time robotic indoor 3D mapping system using duel 2D laser range finders. Chinese Control Conference. IEEE

[25] Petr, O., Michal, K., Pavel, M., et al (2016). Calibration of Short Range 2D Laser Range Finder for 3D SLAM Usage. Journal of Sensors, pp. 1-13.

\section{NOMENCLATURE}
NDT
DIC
PLC
ROI
$\left\{\mathrm{O}_{\mathrm{A}}-\mathrm{X}_{\mathrm{A}} \mathrm{Y}_{\mathrm{A}} \mathrm{Z}_{\mathrm{A}}\right\}$
$\{\mathrm{O}-\mathrm{XYZ}\}$

\author{
Non-Destructive Testing \\ Digital Image Correlation \\ Programmable Logic Controller \\ Region of Interesting \\ Coordinate system of laser ranging sensor position. \\ Coordinate system of the image acquired by laser scanning.
}


$\mathrm{t}(\mathrm{s})$

$\mathrm{v}(\mathrm{m} / \mathrm{s})$

$\alpha$

$\mathrm{S}\left(\mathrm{x}_{\mathrm{a}}, \mathrm{x}_{\mathrm{b}}, \mathrm{x}_{\mathrm{c}}\right)$

$\theta$

$\mathrm{P}_{2} \mathrm{P}_{1}$

$\mathrm{D}$

M4

$1200 \mathrm{rpm}$

C7\&G1610

Gocator-2080

$40 \mathrm{X}$
Movement time of the sensor

Speed of sensor movement

Angle between the laser emitted by the transmitting end and $\mathrm{X}_{\mathrm{A}}$

Coordinate of the intersection with the object

Angle between the emitted light and the reflected light

Plane distance

Height difference

Screw type

Speed of stepper motor

Type of ball screw

Model of laser sensor

40 times magnification 